\title{
SUMOylation is Required for ERK5 Nuclear Translocation and ERK5-Mediated Cancer Cell Proliferation
}

\author{
Tatiana Erazo, Sergio Espinosa-Gil, Nora Diéguez-Martínez, Néstor Gómez and Jose M \\ Lizcano* \\ Institut de Neurociències and Departament de Bioquímica i Biología Molecular. Facultat de Medicina. \\ Universitat Autònoma de Barcelona, 08193 Bellaterra, Barcelona, Spain. erazoani@mskcc.org (T.E.); \\ sergio.espinosa@uab.cat (S.E-G.); nora.dieguez@uab.cat (N.D-M.); nestor.gomez@uab.cat (N.G.); \\ josemiguel.Lizcano@uab.es (JM.L.) \\ * Correspondence, Jose M Lizcano: JoseMiguel.Lizcano@uab.es. Tel.: +34-93-5813076
}

\begin{abstract}
The MAP kinase ERK5 contains an N-terminal kinase domain and a unique C-terminal tail including a nuclear localization signal and a transcriptional activation domain. ERK5 is activated in response to growth factors and stresses, and regulates transcription at the nucleus by either phosphorylation or interaction with transcription factors. MEK5-ERK5 pathway plays an important role regulating cancer cell proliferation and survival. Therefore, it is important to define the precise molecular mechanisms implicated in ERK5 nucleo-cytoplasmic shuttling. We previously described that the molecular chaperone Hsp90 stabilizes and anchors ERK5 at the cytosol, and that ERK5 nuclear shuttling requires Hsp90 dissociation. Here, we show that MEK5 or Cdc37 overexpression -mechanisms that induce nuclear ERK5- induced ERK5 SUMO-2 modification at residues Lys6/Lys22 in cancer cells. We also show that overexpression of the SUMO protease SENP2 completely abolished endogenous ERK5 nuclear localization in response to EGF stimulation. Furthermore, mutation of these SUMO sites abolished the ability of ERK5 to translocate to the nucleus and to promote prostatic cancer PC-3 cell proliferation. These results allow us to propose a more precise mechanism: in response to MEK5 activation, ERK5 SUMOylation favors the dissociation of Hsp90 from the complex, allowing ERK5 nuclear shuttling and activation of transcription.
\end{abstract}

Keywords: MAP kinase, ERK5, Bmk1, SUMO, nuclear translocation, transcription, cell proliferation, cancer, Hsp90, Cdc37.

\section{Introduction}

The extracellular-signal-regulated kinase 5 (ERK5, also called Big MAP Kinase-1 (BMK1) is the most structurally divergent member of the mitogen-activated protein kinase (MAPK) family. ERK5 protein contains a N-terminal kinase domain that shares 50\% identity with ERK1/2 [1], and a unique Cterminal tail, with no homology with any other protein, containing a nuclear localization signal (NLS) and a transactivation domain (TAD) [2]. ERK5 activates transcription by either phosphorylation of transcription factors such as MEF2 [3], Sap1 [4] or c-Myc [5,6], or though the TAD domain in a kinaseindependent mechanism.

ERK5 is ubiquitously expressed in numerous tissues and is activated in response to growth factors and to different forms of stress. ERK5 is activated by direct phosphorylation of the TEY motif within the activation loop of the kinase domain by the MAPK kinase 5 (MEK5) [7]. MEK5 is the only upstream kinase described for ERK5 and, therefore MEK5-ERK5 constitute a unique signaling axis to control cell differentiation, proliferation and survival [8]. Importantly, ERK5 plays a major role in 
regulating cell cycle progression and proliferation in response to a wide range of growth factors such as EGF [3], VEGF [9], FGF-2 [10], NGF [11], CSF-1 [12], IL-6 [13] and PDGF [14]. Mechanistically, ERK5 controls the G1/S phase transition via transcriptional regulation of cyclin D1 [15]. Active ERK5 phosphorylates and activates MEF2A, C and D transcription factors $[3,5,16]$, promoting c-Jun- and c-Fos-mediated expression of cyclin D1 required for cell proliferation $([4,17])$. Moreover, ERK5 also allows cancer cells to evade cell cycle suppressors by impairing the expression of the CDK inhibitors p15, p21 and p27 [18,19].

In addition to the well stablished role of ERK5 in sustaining proliferative signals and evading growth suppression, numerous studies have underlined an implication of ERK5 in almost all hallmarks of cancer (recently reviewed in [20]). For instance, and among others, ERK5 pathway is associated with increased metastatic activity in prostate [21] and hepatocarcinoma [19] cancers, to elevated growth of breast cancer cell overexpressing ErbB2/Her2 receptor [22], or to chemoresistance of breast cancer [23] or acute myeloid leukemia cells [24]. Consequently, genetic and pharmacologic manipulation of the MEK5-ERK5 pathway has a deep impact in cell and tumor viability and, therefore this pathway has been proposed as a new target to tackle different solid and blood cancers.

The subcellular distribution of ERK5 is critical for regulating its role in cancer proliferation and survival. Indeed, nuclear ERK5 correlates with poor prognosis in aggressive prostatic carcinoma $[21,25]$ and in hepatocellular carcinoma [19], and also confers resistance to TRAIL-induced apoptosis in breast cancer models [26]. Thus, it is important to define the precise molecular mechanisms implicated in ERK5 nucleo-cytoplasmic shuttling. Initially, it was proposed that ERK5 N-terminal and C-terminal halves interact forming either a putative NES or a domain that binds a cytoplasmic anchor protein [27]. After activation, the autophosphorylation of the C-terminal tail (at residues S421, S433, S496, S731, and T733; [28]) would disrupt the intramolecular interaction, resulting in the exposition of the NLS and nuclear translocation of ERK5 [27]. Later, we described that the chaperone Hsp90 acts as the cytosolic anchor protein. Indeed, cellular ERK5 interacts with chaperones Hsp90 and Cdc37 in basal conditions, which stabilize and retain ERK5 at the cytosol [29]. In a canonical mechanism, and in response to mitogenic factors, MEK5-mediated ERK5 activation results in phosphorylation of the C-term tail, Hsp90 dissociation and nuclear shuttling. In a non-canonical mechanism, the overexpression of Cdc37 (as it happens in several cancers [30]) also induces Hsp90 dissociation and nuclear translocation of a catalytically inactive -but transcriptionally active- form of ERK5 [29].

Small Ubiquitin-related Modifier (SUMO) is a type of post-translational modification that plays an important role in regulating protein activity, stability, interactions with other proteins and subcellular localization, among others [31]. SUMO is covalently linked to lysine residues in the target proteins through a process that is mechanistically analogous to ubiquitination. The SUMO pathway begins with a SUMO-activating enzyme (SAE1/SAE2), which induces the activation of SUMO C-terminal domain, and transfers activated SUMO to the SUMO-conjugating enzyme (SUMO E2 ligase) Ubc9 [32]. Finally, one of several SUMO-protein E3 ligases PIAS (protein inhibitor of activated STAT) promotes the covalent binding between the lysine of the protein target and the glycine-glycine dipeptide at the SUMO C-terminus. SUMO modification is a reversible and transient protein modification, and it is de-conjugated from targets by specific SUMO isopeptidases called sentrinspecific proteases (SENP) [33].

In this study, we aimed to investigate the role of SUMO modification in the nuclear import of ERK5. We show that ERK5 becomes SUMOylated in response to EGF-induced MEK5 phosphorylation and Cdc37 overexpression, and that SUMOylation is a necessary event, but not sufficient per se, for ERK5 nuclear shuttling. 


\section{Results}

\subsection{MEK5 activity and Cdc37 overexpression induce ERK5 SUMOylation at Lys6 and Lys22.}

It has been previously reported that advanced glycation end products (AGE) and $\mathrm{H} 2 \mathrm{O} 2$ induce ERK5 SUMOylation at residues Lys6 and Lys22 in cultured endothelial cells and cardiomyocytes, and that this posttranslational modification modulates the transcriptional activity of ERK5 [34,35]. However, the authors did not explore the relevance of SUMO modification in ERK5 cellular localization. Given the role of SUMOylation in nuclear translocation of targeted proteins, we decided to study the effect of this posttranslational modification on the subcellular localization of ERK5 in cancer cells. To do so, we have used two different paradigms for ERK5 nuclear shuttling: active MEK5 (canonical mechanism) and Cdc37 (non-canonical mechanism) overexpression [36].

To investigate whether SUMO modification is required for ERK5 nuclear translocation, we performed in vivo SUMOylation assays. We transiently overexpressed in HEK293T cells His-tagged SUMO2 and the E2-conjugating enzyme Ubc9, alone or in combination with ERK5 wild type or an ERK5 SUMO-deficient mutant (K6/22R, in which the two SUMO sites Lys6 and Lys22 have been replaced by Arg). To promote ERK5 nuclear translocation, cells were transfected with vectors encoding either for a constitutive active form of MEK5 (MEK5DD, in which residues Ser311 and Ser315 have been replace by Asp) or Cdc37. Levels of SUMOylated ERK5 were determined by pulling down His-SUMO2 from lysates with Ni2+-NTA-agarose beads and immunoblotting for ERK5. Figure 1a shows that overexpression of MEK5DD or Cdc37 resulted in slow migrating species of ERK5. SUMO2 contains an acceptor Lys (K11) that allows the formation of polySUMO chains [32]. Therefore, the high-molecular-weight smear of bands corresponded to SUMOylated ERK5 species. In contrast, ERK5 mutant K6/22R did not show slow migrating species, confirming that Lysines 6 and 22 are the residues that covalently bind SUMO (Fig 1A). Parallel experiments overexpressing a mutant form of ERK5 that lacks C-terminal tail (ERK5(1-490)) showed SUMO conjugation in response to MEK5DD or Cdc37 overexpression, indicating that the C-term tail is not required for the interaction with the putative SUMO E3 ligase (Figure 1b). These results demonstrate for the first time that MEK5 activity or Cdc37 overexpression induce ERK5 SUMOylation in Lys6 and Lys22 residues.
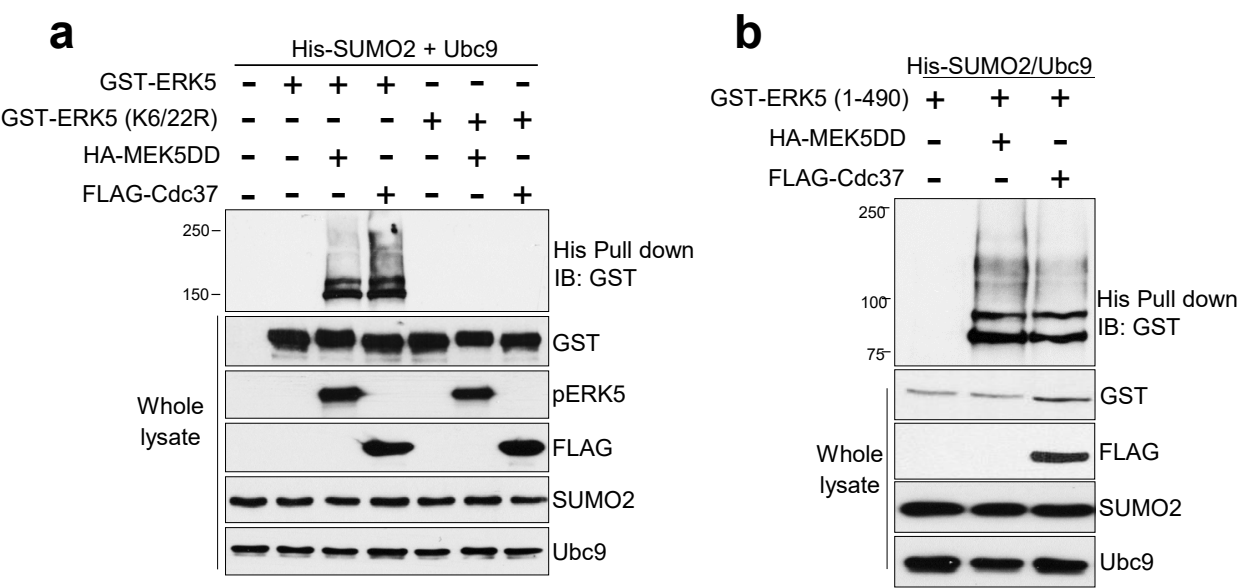

Figure 1. MEK5 phosphorylation or Cdc37 overexpression induce ERK5 SUMOylation. (a), HEK293T cells we co-transfected with His-tagged SUMO2 and Ubc9 and the indicated combinations of GST-tagged ERK5 (wild type or SUMO deficient K6/22R mutant), HA-tagged MEK5DD (constitutively active) and FLAG-tagged Cdc37, as described in Experimenal Procedures. After lysing the cells with denaturing buffer containing NEM, SUMOylated ERK5 was affinity purified using $\mathrm{Ni}^{2+}$ agarose beads and detected by immunoblotting with anti-GST antibody. Levels of overexpressed proteins and phosphorylated ERK5 at the T-loop are shown using the corresponding antibodies. (b), SUMOylation analysis of ERK5 N-terminal half. Experiments were performed as in A, using a mutant form of ERK5 that encodes for the aa 1-490 which contains the kinase domain (aa 54-346). Similar results were obtained in three separate experiments. 
2.2. SUMOylation is required for ERK5 nuclear translocation in response to MEK5-mediated activation or to Cdc37 overexpression.

To study the role of SUMOylation in ERK5 nuclear translocation we used human prostatic adenocarcinoma PC-3 cells, a good cellular model to study the cytoplasmic-nuclear trafficking of ERK5 [21,29]. We overexpressed either ERK5 or the ERK5-SUMO deficient mutant ERK5-K622/R, and ERK5 subcellular localization was monitored by fluorescence microscopy. As expected, overexpressed ERK5-WT showed cytosolic localization in basal conditions (as it does endogenous ERK5), and nuclear staining in response to overexpression of MEK5DD or Cdc37 (Figure 2a). In contrast, the SUMO-deficient mutant did not translocate to the nucleus in response to overexpression of any of these two proteins. Furthermore, $18 \mathrm{~h}$ incubation of cells with the inhibitor of nuclear protein export Leptomycin B resulted in nuclear accumulation of ERK5-WT, but still did not affect cytosolic localization of ERK5-SUMO deficient mutant (Figure 2b). These results show for first time that SUMOylation is required for ERK5 nuclear translocation in response to MEK5-mediated activation or to Cdc37 overexpression.

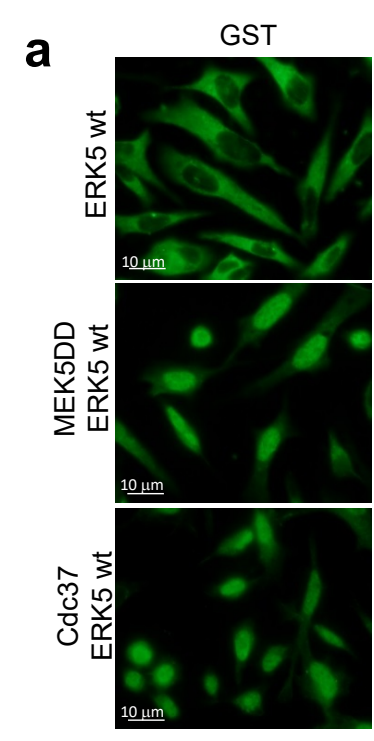

b

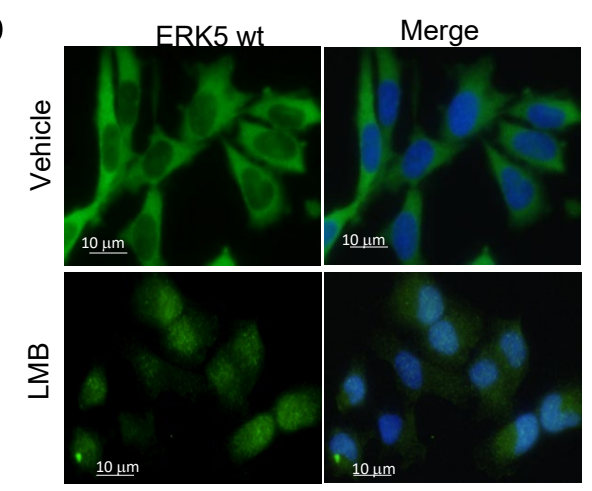

Merge

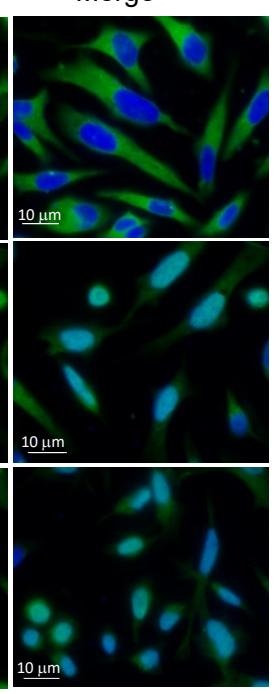

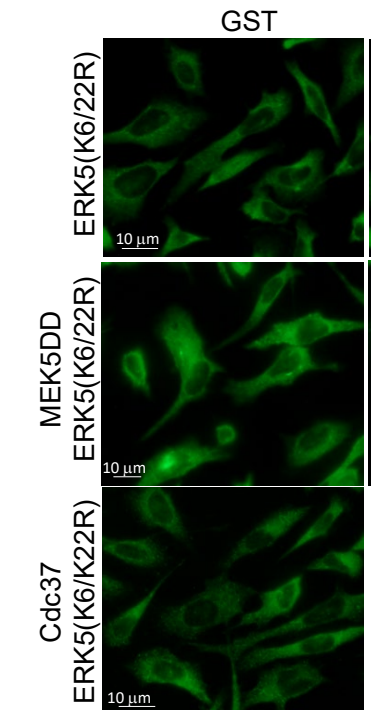
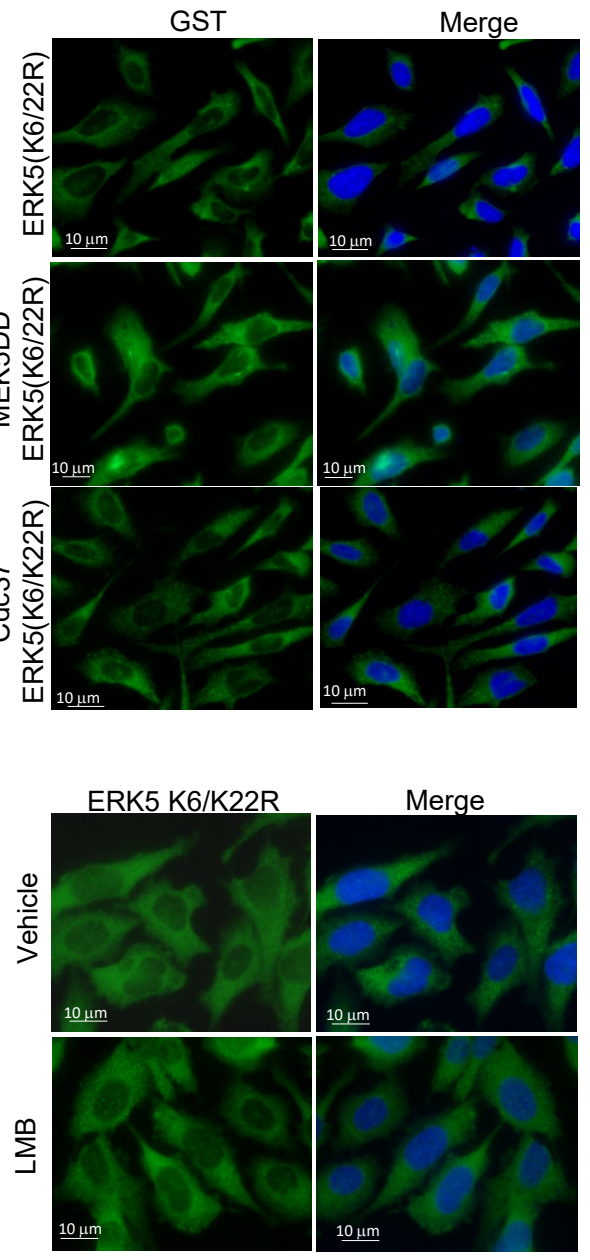

Figure 2. ERK5 requires SUMO modification for nuclear translocation. Immunofluorescence microscopy. (a), PC-3 cells were transfected with either GST-tagged ERK5 (wild type or K6/22R SUMO deficient mutant) and MEK5DD or Cdc37 plasmids. After 24 hours, cells were fixed with paraformaldehyde and immunofluorescent staining for ERK5 (green) using anti-GST antibody. Nuclei were stained with Hoechst (blue). (b), PC-3 cells were transfected with either GST-tagged ERK5 WT or K6/22 mutant, left alone or treated with the nuclear export inhibitor leptomycin B (LMB) for $18 \mathrm{~h}$, and stained as in A. Similar results were obtained in three independent experiments. Scale bars, $10 \mu \mathrm{m}$ 
We performed analogous experiments to study the subcellular localization of endogenous ERK5. Several authors have previously shown that Epidermal Growth Factor EGF induces ERK5 activation and nuclear translocation in different cancer cells $[3,19,27]$. As expected, PC-3 cells showed cytosolic ERK5 in basal conditions, and nuclear localization in response to short-time EGF stimulation (15 min) (Figure 3a). Protein SUMOylation could be reverted by the Sentrin-specific proteases (SENPs), which break the linkage between the N-terminal Gly of SUMO and the Lys of the targeted protein $[33,38]$. Thus, we next investigated the role of ERK5 SUMOylation in EGF-induced nuclear translocation by reverting its SUMOylation, using the SUMO protease SENP2, the isoform involved in ERK5 deSUMOylation [37]. Overexpression of SENP2 in PC-3 cells prevented endogenousERK5 nuclear translocation in response to EGF-stimulation or Cdc37 overexpression (Figure $3 \mathrm{~b}$ and $3 \mathrm{c}$, respectively), indicating that SUMOylation is also necessary for nuclear translocation of endogenous ERK5.
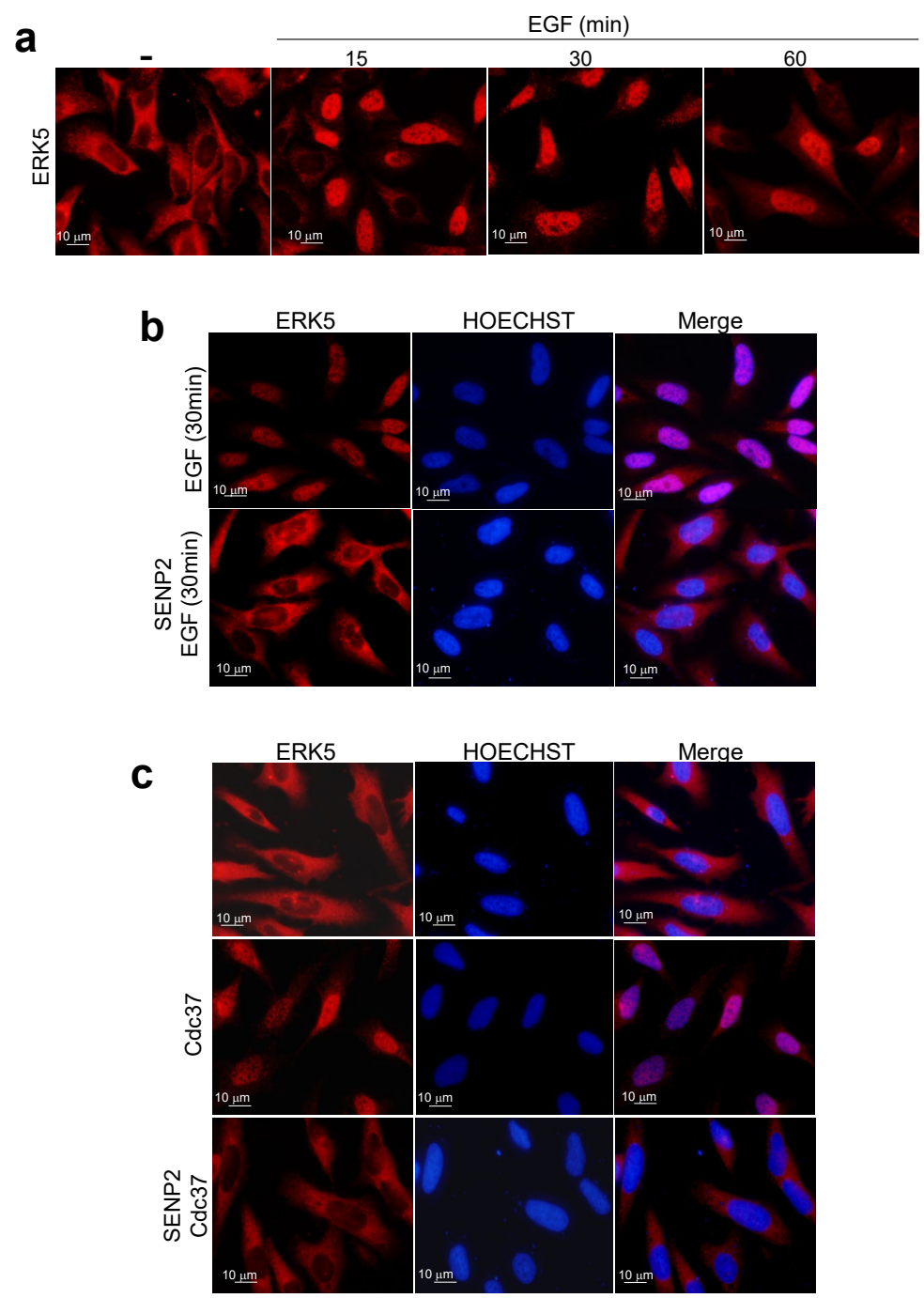

Figure 3. Overexpression of SUMO peptidase SENP2 inhibits nuclear translocation of endogenous ERK5. (a), ERK5 nuclear translocation in response to EGF. PC-3 cells were starved prior to stimulation with $50 \mathrm{ng} / \mathrm{ml}$ EGF. At the indicated times, cells were fixed and immunofluorescent stained for ERK5. (b), Effect of SENP2 on EGF-mediated ERK5 nuclear translocation. Transfected cells with empty vector (control) o vector encoding for SUMO protease SENP2. $36 \mathrm{~h}$ later cells were starved for $16 \mathrm{~h}$ prior stimulation with $50 \mathrm{ng} / \mathrm{ml}$ for $30 \mathrm{~min}$. Cells were immunostained for endogenous ERK5 (red), and nuclei (Hoechst, blue). (c), Effect of SENP2 on Cdc37-mediated ERK5 nuclear translocation. Transfected cells with empty vector (control) or vector encoding for Cdc37 alone or in combination with a vector encoding SENP2 were fixed and ERK5 visualized by immunostaining (red). Similar results were obtained in three independent experiments. Scale bars, $10 \mu \mathrm{m}$ 


\subsection{SUMO modification does not affect ERK5 kinase activity or its activation by MEK5.}

Next, we explored whether SUMOylation affects ERK5 kinase activity. To do so, we performed in vitro kinase assays using HEK293 cell lysates overexpressing GST-tagged ERK5, alone or in combination with active MEK5, and 32P-ATP and PIMtide peptide as substrates [29]. MEK5DD overexpression resulted in a robust increase of kinase activity of both ERK5-WT and SUMO-deficient mutant, (Figure 4a). Moreover, ERK5 WT and kinase-inactive mutant (D200A) resulted SUMOylated in response to overexpression of active MEK5 or Cdc37 (Figure 4b). These results indicate that not only ERK5 kinase activation does not require SUMOylation, but also that SUMOylation is independent of ERK5 kinase activity. Our observations are in agreement with those reported by Woo et al., who showed that SUMOylation in response to $\mathrm{H} 2 \mathrm{O} 2$ or AGE does not affect ERK5 kinase activity [34]. Interestingly, we observed that the ERK5 kinase-inactive mutant did not translocated to the nucleus in response to MEK5 overexpression (Figure 4c), indicating that SUMOylation is not sufficient per se to induce ERK5 nuclear translocation.
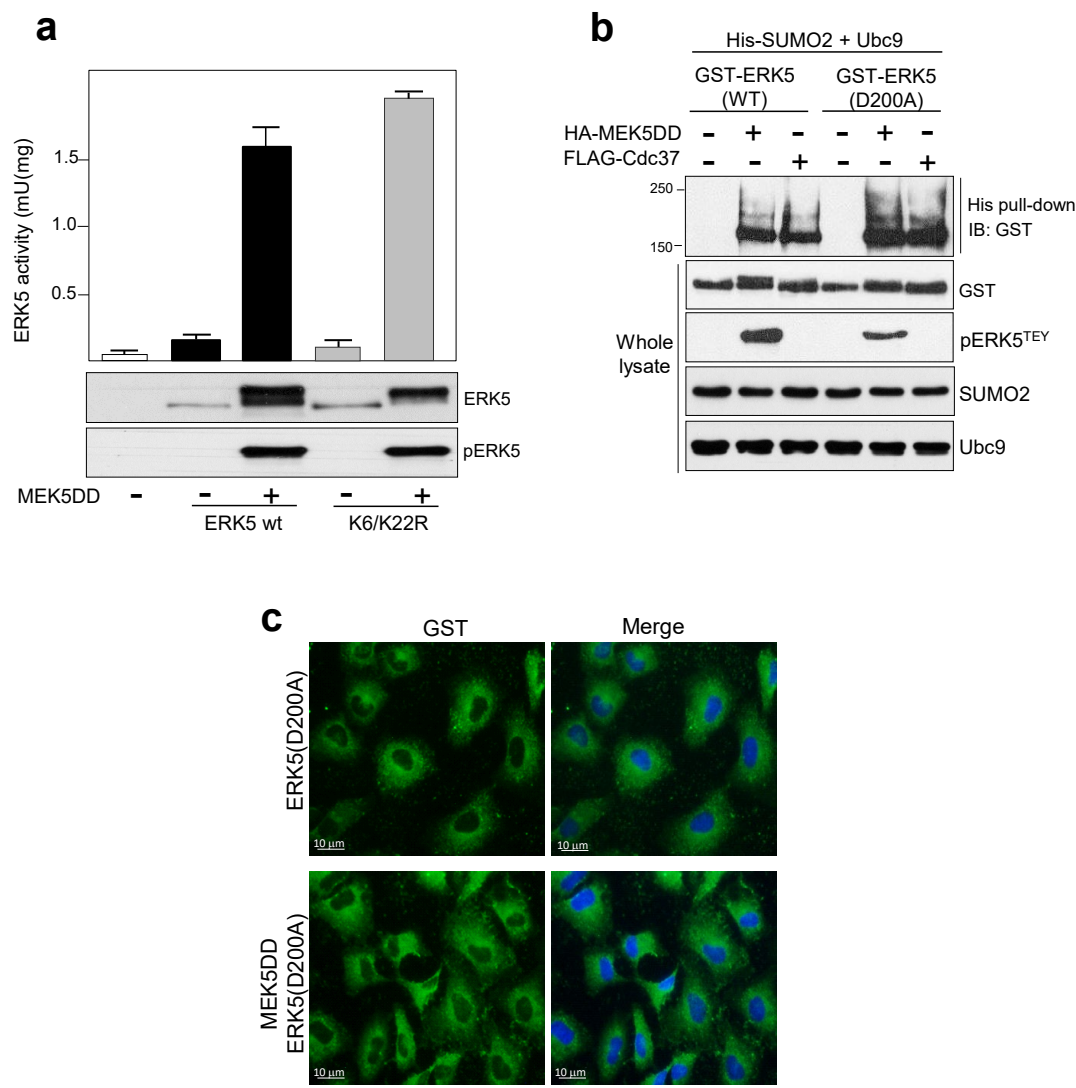

Figure 4. ERK5 SUMOylation is not necessary for ERK5 kinase activity. (a) HEK293T cells overexpressing GST-tagged ERK5 (wild type or SUMO deficient K6/22R mutant), alone or in combination with MEK5DD, were lysed and GST-ERK5 protein was affinity purified and assayed for kinase activity as described in the Methods Section. Activity data are the mean \pm SD of two independent experiments, each performed in triplicate. Cell lysates were also immunoblotted for ERK5 (anti-GST antibody) and for phosphorylated ERK5 (pERK5). (b) HEK293T cells were co-transfected with Histagged SUMO2 and Ubc9, and the indicated combinations of GST-tagged ERK5-D200A (kinase inactive), HA-tagged MEK5DD (constitutively active) and FLAG-tagged Cdc37. After lysing the cells with denaturing buffer containing NEM, SUMOylated ERK5 was affinity purified using $\mathrm{Ni}^{2+}$-agarose beads and detected by immunoblotting with anti-GST antibody. Levels of overexpressed proteins and phosphorylated ERK5 at the T-loop are shown using the corresponding antibodies. Similar results were obtained in three independent experiments. (c), Immunofluorescence microscopy. PC-3 cells were transfected with GST-tagged ERK5 (wild type or kinase-inactive mutant D200A) and MEK5DD plasmids. After 24 hours, cells were fixed with paraformaldehyde and immunofluorescent staining for ERK5 (green) using anti-GST antibody. Nuclei were stained with Hoechst (blue). 


\subsection{SUMOylation is required for ERK5 co-transcriptional activity and cell proliferation.}

Nuclear ERK5 acts as an activator of different transcription factors, such as MEF2 or AP-1 transcriptional complex [17]. Given our results showing that ERK5 nuclear translocation requires SUMOylation, we undertook the corresponding transcriptional assays to monitor AP-1 transcriptional activity in PC-3 cells overexpressing ERK5 WT or SUMO-deficient mutants, in a luciferase-based reporter assay. As previously reported [29], active MEK5 or Cdc37 overexpression resulted in enhanced ERK5-mediated AP-1 transcriptional activity. In turn, the SUMO-deficient mutant had negligible effect on AP-1 transcriptional activity in response to MEK5 or Cdc37 overexpression (Figure 5a), indicating that ERK5 SUMOylation is necessary for ERK5-mediated AP1 transcriptional activity.
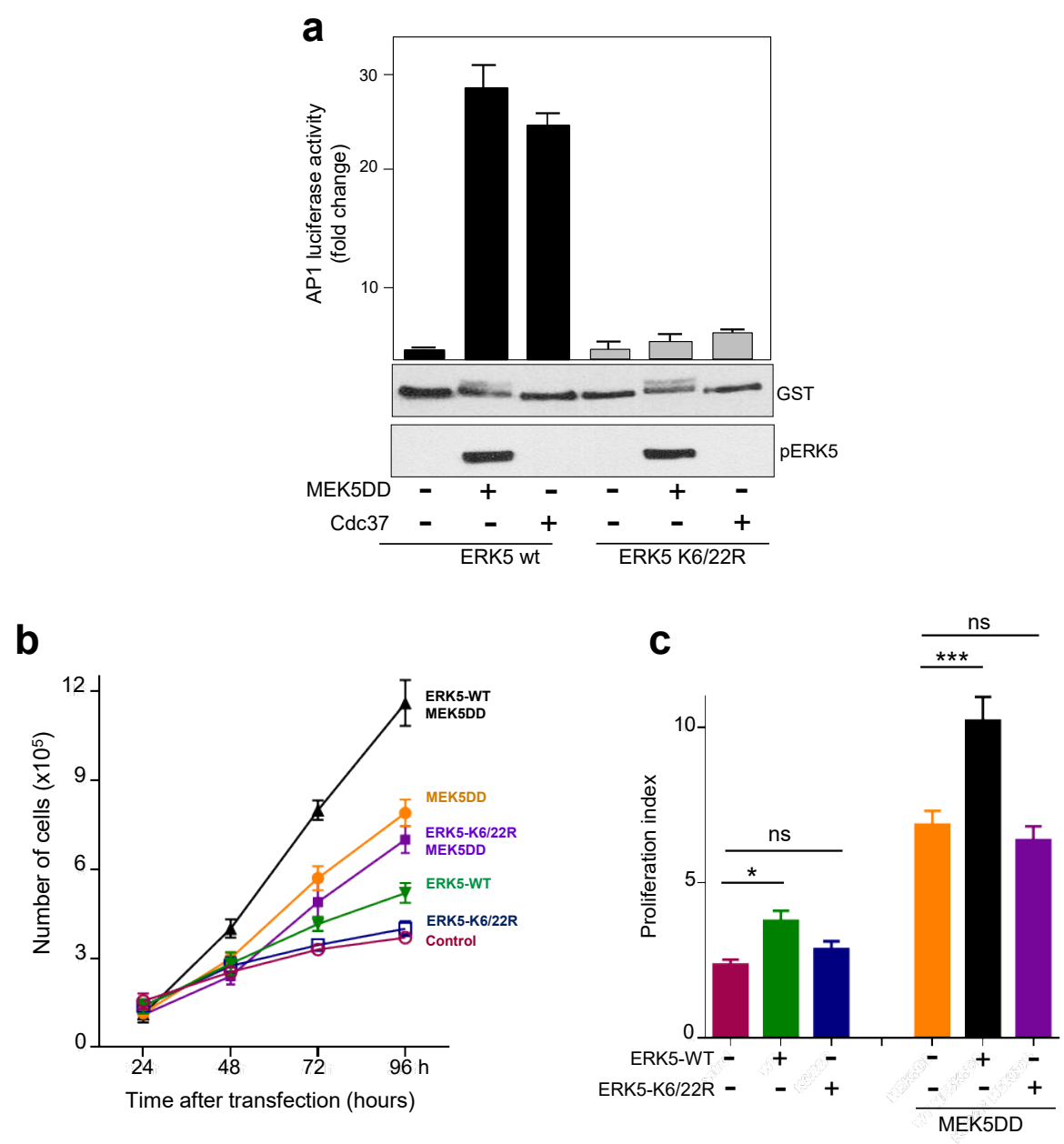

Figure 5. ERK5 SUMOylation is necessary for transcriptional activity and cell proliferation. (a), SUMO modification is required for ERK5-mediated AP-1 transcriptional activity. pAP-1 luciferase reporter and pRL-CMV-Renilla plasmids were co-transfected with the indicated plasmids in HeLa cells. 24 later, lysates were subjected to a dual-luciferase assay. Each value is the mean \pm SD of three separate determinations, each performed in triplicate and normalized using the Renilla values. Cell lysates were also immunoblotted for ERK5 (anti-GST antibody) and for phosphorylated ERK5 (pERK5). (b), ERK5 SUMOylation is necessary for ERK5-mediated cell proliferation. PC-3 cell were transfected with plasmids encoding for ERK5-WT, ERK5-K6/22 mutant, MEK5DD, or for ERK5-WT and MEK5DD or ERK5-K6/22R and MEK5DD. At the indicated times, cells were counted as described in Materials and Methods. Left panel shows the corresponding proliferation curves; right panel shows the corresponding proliferation index values, measured as the ratio number cells at day 4 : number of cells at day 1 . Each value is the mean $\pm \mathrm{SD}$ of the results determined for three different transfected cell dishes. Similar results were obtained in two independent experiments. ${ }^{*}, \mathrm{P}<0.05$; ${ }^{* * *}, \mathrm{P}<0.001$. 
Of note, ERK5 plays an important role on the proliferation of cancer cells, through activating transcriptional activity of pro-proliferative factors such as AP-1 complex, among others. Therefore, we next asked whether ERK5 requires SUMO modification to activate cell proliferation. We used PC3 prostatic cancer cells since overexpression of MEK5 or ERK5 results in enhanced proliferation of these cells $[21,25,39]$. In agreement with our results showing that SUMOylation is required for ERK5mediated AP-1 activity, ERK5 overexpression resulted in an increase on PC-3 cell proliferation index, whereas no effect was observed after overexpressing the SUMO-deficient ERK5 mutant. Furthermore, overexpression of ERK5, but not of SUMO-deficient ERK5 mutant, also enhanced cell proliferation induced by active MEK5 (Figure 5b-c). Overall, our results suggest that SUMO modification plays a role in modulating ERK5-mediated cancer cell proliferation, which agrees with reports showing that SUMO modification of key proteins results in enhanced cancer cell proliferation [40].

\subsection{SUMOylation is necessary for Hsp90 dissociation from the ERK5-Cdc37 complex.}

We previously showed that Hsp90 dissociation from the ERK5-Cdc37 complex is a necessary event for ERK5 nuclear translocation, since Hsp90 acts as a cytosolic anchor for ERK5 [29]. Given our results showing that SUMO modification is also necessary for ERK5 shuttling to the nucleus, we next investigated whether this posttranslational modification is also involved in Hsp90 dissociation. To this end, we transiently expressed in HEK293 HA-tagged Hsp90 and GST-tagged ERK5-WT or the ERK5 SUMO-deficient mutant cells, and ERK5 was activated by overexpression of active MEK5 (MEK5DD). Then, we analyzed the presence of these proteins in the affinity purified ERK5 or Hsp90 pellets. As reported before, activation of ERK 5 by MEK 5 resulted in dissociation of Hsp 90 from ERK5 WT (Figure 6). However, overexpression of active MEK5 did not induce the release of Hsp90 from the ERK5 SUMO-deficient complex. These results suggest that SUMO modification is a necessary event for Hsp90 dissociation in response to MEK5 activation.

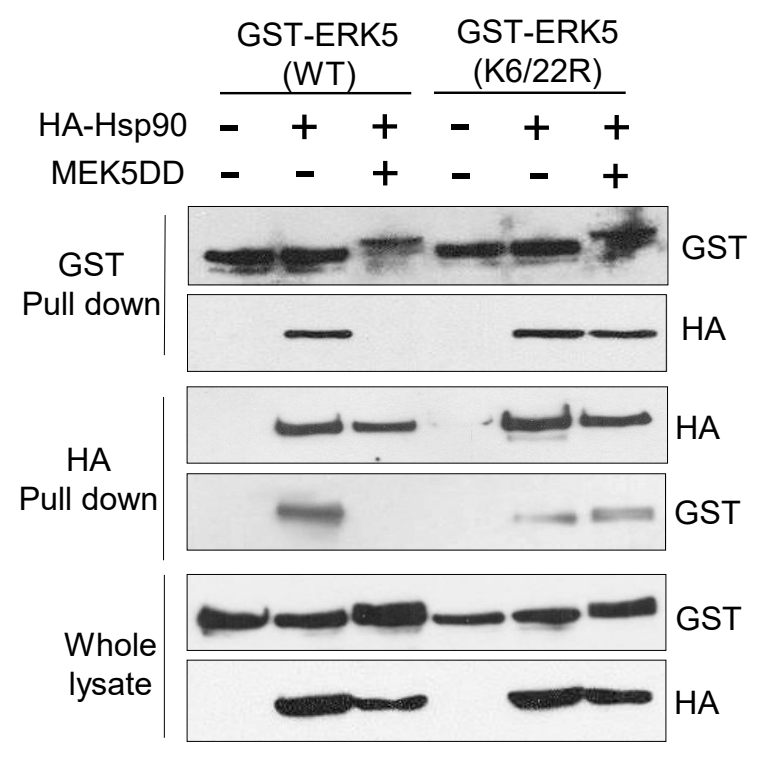

Figure 6. SUMOylation is necessary for Hsp90 dissociation from the ERK5-Cdc37 complex after MEK5 activation. HEK293T cells were transfected with GST-tagged ERK5 (wild type or SUMO deficient K6/22R mutant) alone or in combination with Hsp90 and MEK5DD. Thirty-six h later, cells were lysed and GST-ERK5 or HA-Hsp90 proteins were affinity purified using glutathione-Sepharose or anti-HA agarose resins, respectively. Immune complexes were immunoblotted for ERK5 and Hsp90. Similar results were obtained in three independent experiments. 


\section{Discussion}

ERK5 controls proliferation of cancer cells by acting as a transcriptional co-activator at the nucleus $[3,13,25,41]$. Therefore, it is important to establish the precise molecular mechanisms involved in ERK5 nuclear shuttling, not fully described yet. Previous work showed that ERK5 is SUMOylated at Lys6 and Lys22 in endothelial cells in response to different stresses [34,35], but the authors did not investigate whether this posttranslational modification affects ERK5 transport to the nucleus. Here, we show that MEK5 or Cdc37 overexpression -two mechanisms that induce nuclear ERK5- results in ERK5 SUMOylation at residues Lys6 and Lys22 in cancer cells (Figure 1). We found that SUMO modification does not affect ERK5 kinase activity, but that it is absolutely required for ERK5 nuclear translocation, since SUMO-deficient ERK5 mutant showed a constitutive cytoplasmic localization even in the presence of the nuclear export inhibitor leptomycin B (Figure 2 and 3). Supporting this notion, overexpression of the SUMO protease SENP2 (the enzyme catalyzing ERK5 de-SUMOylation, [37]) completely abolished endogenous ERK5 nuclear localization in response to EGF stimulation (Figure 3).

SUMO modification plays an important role in regulating the subcellular localization of many target proteins. In mammalian cells, protein SUMOylation has been linked to nuclear import [31], and most of the SUMO machinery enzymes are found within the nucleus. That is the case for the IGF-1 receptor, that becomes SUMOylated after IGF-1 stimulation and translocates to nucleus in a SUMO-dependent process [42], the soluble intracellular domain of the ErbB4 receptor [43], or the Polo-like kinase 1 [44], among others. These kinases do not possess an NLS motif, but SUMO modification seems to allow them to interact with nuclear transport proteins, probably facilitating the binding to cargo proteins containing NLS sequences. In the case of ERK5, which possesses a hidden NLS motif, we cannot discard that SUMO modification could facilitate its binding to a cargo protein. However, it is also probably that branched SUMOylation could result in a conformational change of ERK5 into an open conformation that would expose the NLS motif, allowing its transport to the nucleus.

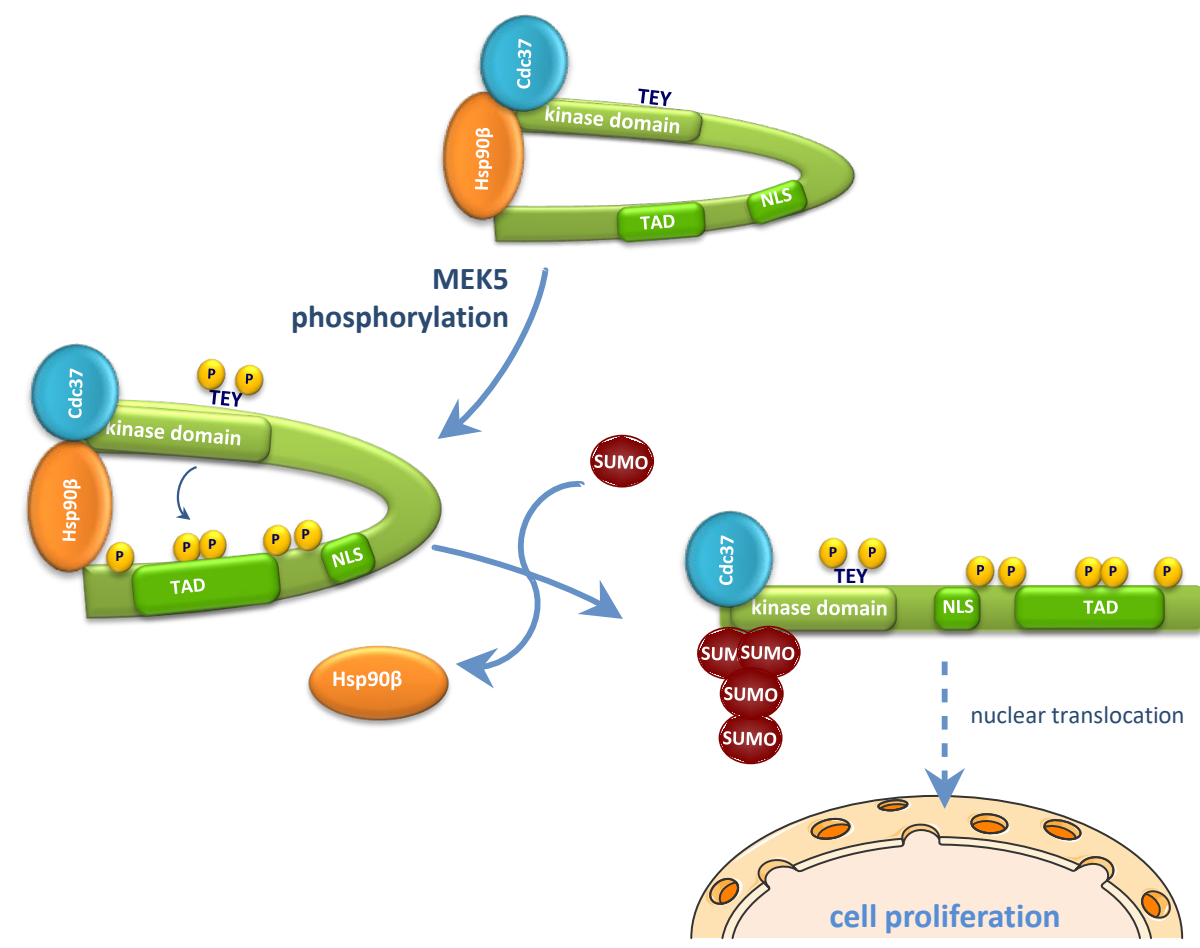

Figure 7. A model for the role of SUMO modification in the mechanism of nucleocytoplasmic shuttling of ERK5. 
The results reported here allow us to propose a more precise mechanism for the nuclear transport of ERK5 in response to MEK5-mediated canonical activation (summarized in Figure 7). This model integrates our previous findings about the role of the cytosolic anchor Hsp90 protein, together with posttranslational modifications such as phosphorylation and SUMOylation. Thus, in basal conditions the C-terminal tail of ERK5 interacts intramolecularly with the N-terminal half, generating a close conformation that enables interaction with the cytosolic anchor protein $\mathrm{Hsp} 90$ and $\mathrm{Cdc} 37 \mathrm{co}-$ chaperone. Mitogens such as EGF induce the activation of the upstream kinase MEK5, which in turn activates ERK5 by phosphorylating the TEY motif. This phosphorylation allows the recruitment of a specific PIAS SUMO E3 ligase (yet to be described), resulting in SUMOylation of ERK5 (branched SUMO chains) at residues Lys6 and Lys22. Of note, our findings show an interplay between phosphorylation and SUMOylation, as described for other proteins (reviewed in [45]). In this model, covalent SUMO modification of ERK5 would facilitate the dissociation of Hsp90 from the kinase domain (Hsp90 is not dissociated from ERK5 SUMO-deficient mutant in response to MEK5 activation, Figure 6). Then, active ERK5 autophosphorylates its C-term tail, inducing a conformational change that results in exposure of the NLS and in the transport of ERK5 to the nucleus. In our model, SUMOylation is a necessary event, but not sufficient per se, for ERK5 nuclear translocation, since the ERK5 kinase inactive mutant (D200A) becomes SUMOylated in response to MEK5 activation (Figure $4 \mathrm{~b}$ ) but it does not translocate to nucleus (Figure $4 \mathrm{c}$ ).

Our results indicate that SUMOylation is necessary for the transcriptional activity of ERK5, since SUMO-deficient mutant did not show AP-1 transcriptional activity (Figure 5a). Nevertheless, this can lead to misinterpretation of the real role of SUMO modification on ERK5 transcriptional activity. Given the fact that SUMOylation is required for ERK5 nuclear import and that ERK5 needs to be at the nucleus to act as transcriptional co-activator, it can be expected that SUMO-deficient mutant will not show transcriptional activity. However, it cannot be ruled out that ERK5 could be deSUMOylated by the SENP2 protease at the nucleus. SENP2 localizes at the internal side of the nuclear pore via interaction with the nuclear pore protein NUP153 [46], and it is involved in de-SUMOylation of proteins after nuclear entry [42]. Supporting this, we found that SENP2 specifically repressed endogenous ERK5 nuclear translocation induced by EGF stimulation (Figure 3c). Thus, we cannot discard that ERK5 is subjected to a dynamic SUMOylation/de-SUMOylation cycle at the nuclear pore and therefore, that nuclear de-SUMOylation is required for ERK5 to act as a transcriptional coactivator. In this regard, it has been previously shown in non-tumoral endothelial cells that advanced glycation end products (AGE) and $\mathrm{H} 2 \mathrm{O} 2$ induce ERK5 SUMOylation at Lys6 and Lys22, resulting in impaired ERK5-mediated transcriptional activity of MEF2 transcription factor in response to MEK5 overexpression [34,35]. Thus, these observations suggest that non-SUMOylated ERK5 is the specie that interacts with transcription factors. Further experiments will be required to address this important question.

Finally, and in line with data presented here indicating that SUMOylation is required for ERK5 nuclear translocation, we show that SUMO modification is necessary for ERK5-induced AP-1mediated proliferation of prostatic adenocarcinoma PC-3 cells (Figure 5b-c). Expression of key components of the SUMO machinery (such as SUMO1 E1 activating enzyme, E2 conjugation enzyme Ubc9 and some PIAS SUMO E3 ligases) is enhanced in many human cancers, and it correlates with poor prognosis (reviewed in [47]). Of relevance for our work, it has been reported elevated expression levels of Ubc9 and PIAS1 enzymes in human prostate adenocarcinoma [48,49]. Given the fact that nuclear ERK5 is associated with proliferation, invasion and bad prognosis in prostate cancer [21,25], it is likely that this scenario of enhanced SUMOylation dynamics could result in favoring ERK5 SUMOylation and, therefore ERK5 nuclear localization. In this context, it will be interesting to explore if targeting ERK5 SUMOylation could results in an effective approach to tackle prostate cancer, as well other cancers showing nuclear ERK5 and enhanced SUMO machinery players. 


\section{Materials and Methods}

\subsection{Materials}

N-ethyl-maleimide, Ni2+-NTA-agarose, anti-HA-agarose, Epidermal growth factor, leptomycin B and phosphocellulose paper p81 were from Sigma. Polyethylenimine (PEI) was from Polysciences, and protein-G-Sepharose, glutathione-Sepharose were from GE-Healthcare. [ $\gamma$-32P]-ATP was from Perkim-Elmer.

\subsection{Antibodies}

The polyclonal antibodies anti-ERK5 and anti-phospho ERK5-pT218EY220 were from Cell Signaling Technology. Anti-GST, anti-FLAG and anti-HA monoclonal antibodies were from Sigma; anti-Cdc37 and anti-ERK5 antibody (immunofluorescence experiments) from Santa Cruz Biotechnology; antiHsp90 $\beta$ from Merck Biosciences; and the anti-SUMO antibody from Invitrogen. The anti-Ubc9 antibody was a gift of Dr. R. Hay (College of Life Sciences, University of Dundee, Dundee, UK).

\subsection{DNA constructs}

Recombinant DNA procedures were performed using standard protocols. The pEBG-2T vectors encoding for GST-tagged human ERK5 full length (WT), kinase inactive mutant (D200A) or Nterminus (aa 1-490) were a gift from Dr. P. Cohen (MRC Protein Phosphorylation Unit, Dundee, UK) [50], and they have been described before [29]. The vector containing Human ERK5 SUMO-deficient K6/22R was from Dr J-I Abe (University of Rochester, NY) and it was used to generate N-term GST tagged ERK5 K6/22R mutant in a pEBG-2T vector. The pCMV plasmid encoding HA-tagged MEK5DD (constitutively active) was from Dr. E. Nishida (Kyoto University, Japan, [27]), the pCDNA3 vector encoding FLAG-tagged Cdc37 was a gift of Dr. Scheidereit (Center for Molecular Medicine, Berlin, Germany, [51]), and the pcDNA3.1 vector encoding HA-tagged Hsp90 $\beta$ was from Dr. Papapetropoulos (University of Athens, Greece, [52]). pCDNA3 vectors encoding Ubc9 and 6xHistagged SUMO2 were from Dr. R. Hay (College of Life Sciences, University of Dundee, Dundee, UK). AP1-luciferase vector was purchased from Stratagene, and pRL-CMV-Renilla from Promega.

\subsection{Cell culture, transfection and lysis}

Cells were cultured at $37^{\circ} \mathrm{C}$ under humidified air (5\% CO2). Human HEK293, HeLa and PC-3 cells were cultured in Dulbecco's Modified Eagle Medium (DMEM) medium supplemented with 10\% FBS and antibiotics. Cells were transfected using polyethylenimine as described before [53]. Unless otherwise stated, cells were lysed in ice-cold lysis buffer $(50 \mathrm{mM}$ Tris- $\mathrm{HCl}, \mathrm{pH} 7.5,1 \mathrm{mM}$ EGTA, $1 \mathrm{mM}$ EDTA, $1 \%(\mathrm{w} / \mathrm{v}) \mathrm{NP}-40,1 \mathrm{mM}$ sodium orthovanadate, $10 \mathrm{mM}$ sodium- $\beta$-glycerophosphate, $50 \mathrm{mM}$ sodium fluoride, $5 \mathrm{mM}$ sodium pyrophosphate, $0.27 \mathrm{M}$ sucrose, $0.1 \%$ (vol/vol) 2-mercaptoethanol and complete protease inhibitor cocktail). Lysates were centrifuged at $12,000 \mathrm{~g}$ for $12 \mathrm{~min}$ at $4^{\circ} \mathrm{C}$, and supernatants stored at -20@C. Protein concentration was determined by the Bradford method [54].

\subsection{Immunofluorescence microscopy}

Cells grown on polylysine-coated cover slips were fixed with $4 \%$ paraformaldehyde for 20 min, and mounted in medium with Hoechst 33259 for DNA staining. To monitor endogenous ERK5, fixed cells were processed as described before [29] using anti-ERK5 antibody (C-term, Santa Cruz) and the corresponding fluorescent-labelled secondary antibody and Hoechst 33259. Cells were visualized by fluorescence microscopy, in a Nikon Eclipse 90i epifluorescence microscope.

\subsection{Immunoprecipitation and Immunoblotting}


$10 \mu \mathrm{l}$ protein G-Sepharose beads bound to $2 \mu \mathrm{g}$ of the corresponding antibody were incubated with $0.5 \mathrm{mg}$ of cell lysate for $2 \mathrm{~h}$ at $4^{\circ} \mathrm{C}$. The immunoprecipitates were washed twice with lysis buffer containing $0.15 \mathrm{M} \mathrm{NaCl}$, twice with buffer A (50 mM Tris- $\mathrm{HCl} \mathrm{pH} 7.5,0.1 \mathrm{mM}$ EGTA, $0.1 \%$ 2mercaptoethanol) and the immune complexes eluted in 2x sample buffer. GST-, HA- and FLAGtagged overexpressed proteins were immunoprecipitated as described above, using the appropriate resin. Immunoblotting was performed as described previously [55].

\subsection{In vivo ERK5 SUMOylation assay}

We followed the method described by Tatham et al. [56]. Ten $\mathrm{cm}$ dishes of HEK293 cells overexpressing His-tagged SUMO2, the E2 ligase Ubc9 and the indicated form of ERK5 were resuspended in $5 \mathrm{ml}$ of buffer 1 (6 $\mathrm{M}$ guanidinium- $\mathrm{HCl}, 10 \mathrm{mM}$ Tris $100 \mathrm{mM}, 100 \mathrm{mM}$ $\mathrm{Na} 2 \mathrm{HPO} / \mathrm{NaH} 2 \mathrm{PO} 4$ buffer $\mathrm{pH}$ 8), sonicated for $1 \mathrm{~min}$ and centrifuged at 5,000 $\mathrm{g}$ for $5 \mathrm{~min}$. The resulting supernatants were incubated with $20 \mu \mathrm{L}$ of Ni2+-NTA-agarose beads for $2 \mathrm{~h}$ at room temperature with rotation. Beads were successively washed as follows: twice with $4 \mathrm{ml}$ of Buffer 1 plus $10 \mathrm{mM}$ 2-mercaptoethanol; three times with $4 \mathrm{ml}$ of buffer $2(8 \mathrm{M}$ urea, $10 \mathrm{mM}$ Tris, $10 \mathrm{mM} 2-$ mercaptoethanol, $100 \mathrm{mM} \mathrm{Na} 2 \mathrm{HPO} 4 / \mathrm{NaH} 2 \mathrm{PO} 4$ buffer $\mathrm{pH}$ 8), twice with $4 \mathrm{ml}$ of buffer 3 (8 M Urea, $10 \mathrm{mM}$ Tris, $100 \mathrm{mM} \mathrm{Na} 2 \mathrm{HPO} 4 / \mathrm{NaH} 2 \mathrm{PO} 4$ buffer $\mathrm{pH}$ 6.3) containing $10 \mathrm{mM}$ 2-mercaptoethanol, once with $1 \mathrm{ml}$ of buffer 3 containing $0.2 \%$ Triton $X-100$, once with $1 \mathrm{ml}$ of buffer 3 containing $0.1 \%$ Triton $\mathrm{X}-100$ and $0.5 \mathrm{M} \mathrm{NaCl}$; and three washes with $1 \mathrm{ml}$ of buffer 3 . Finally, proteins were eluted by incubating the beads with $200 \mathrm{mM}$ imidazole in 5\% SDS, $0.15 \mathrm{M}$ Tris- $\mathrm{HCl} \mathrm{pH} 6.7,30 \%$ (v/v) glycerol, $0.72 \mathrm{M} 2$-mercaptoethanol for $1 \mathrm{~h}$ at $37^{\circ} \mathrm{C}$ with mixing.

\subsection{ERK5 kinase activity assay}

$0.5 \mathrm{mg}$ of HEK293 cell extracts overexpressing different forms of GST-tagged ERK5 and the indicated proteins were incubated for $1 \mathrm{~h}$ at $4^{\circ} \mathrm{C}$ with $10 \mu \mathrm{l}$ of glutathione-Sepharose beads. Beads were then washed twice with lysis buffer containing $0.5 \mathrm{M} \mathrm{NaCl}$, followed by two washes with buffer A. Kinase activity assay was performed in a assay volume of $50 \mu \mathrm{l}$ containing glutathione-Sepharose beads, buffer A, $10 \mathrm{mM}$ magnesium acetate, and $0.1 \mathrm{mM}[\gamma-32 \mathrm{P}]$-ATP $(500 \mathrm{cpm} / \mathrm{pmol})$ and $500 \mu \mathrm{M}$ PIMtide (ARKKRRHPSGPPTA), a bonafide ERK5 peptide substrate [29]. Assays were carried out for $45 \mathrm{~min}$ at $30^{\circ} \mathrm{C}$, terminated by applying the reaction mixture onto p81 paper and the incorporated radioactivity measured as described previously [29]. One milliUnit of activity is the amount of enzyme that catalyzes the phosphorylation of 1 pmol of PIMtide in 1 min.

\subsection{Reporter luciferase assay}

Cells cultured in 12-wells plates were transfected with $650 \mathrm{ng}$ of DNA, which contained $100 \mathrm{ng}$ of AP-1-driven luciferase reporter construct and $50 \mathrm{ng}$ renilla, and the indicated amounts of plasmids. 24 hours later, luciferase activity assay was performed using the Dual-Luciferase kit (Promega), following the manufacturer's instructions.

\subsection{Statistical analysis}

Figures were generated using Microsoft PowerPoint or Adobe Photoshop software. Statistical significance was determined using the Prism 4.0 software, using two-way ANOVA followed for Tukey's test.

Supplementary Materials: none 
Author Contributions: Conception and design: Tatiana Erazo, Nestor Gomez and Jose M Lizcano. Development of methodology: all the authors. Acquisition of data: Tatiana Erazo, Sergio EspinosaGil and Nora Diéguez-Martínez. Analysis and interpretation of data: all the authors. Writing of the manuscript: Jose M Lizcano and Sergio Espinosa-Gil. Funding: Jose M Lizcano. All authors read and approved the final manuscript.

Funding: This work was supported by the grants from Ministerio de Ciencia y Tecnologia BFU200760268/BMC and Ministerio de Economia y Competitividad SAF2015-64237-R, and co-financed by Fondos FEDER [the European Regional Development Fund (ERDF)]. Both are gratefully acknowledged.

Acknowledgments: We thank Elisabet Megías-Roda for technical assistance and Cristina Gutierrez and Neus Ontiveros for tissue culture assistance. We are grateful to the Servei de Genòmica from the UAB.

Conflicts of Interest: The authors declare no conflict of interest.
Abbreviations
AGE Advanced Glycation End products
BMK1 Big MAP kinase 1
Cdc37 Cell division Cycle 37
EGF Epidermal Growth Factor
ERK1/2 Extracellular Signal-Regulated Kinases 1/2
ERK5 Extracellular Signal-Regulated Kinase 5
Hsp90 Heat Shock Protein 90
MAPK Mitogen-activated Protein Kinase
MEK5 Extracellular Signal-Regulated Kinase Kinase 5
NES Nuclear Export Sequence
NGF Nerve growth factor
NLS Nuclear Localization Signal
PDGF Platelet-derived growth factor
PIAS Protein inhibitor of activated STAT
SAE SUMO-activating enzyme
SENP Sentrin Specific Protease
SUMO Small Ubiquitin-like Modifier Protein
TAD Transcriptional Activation Domain
Ubc9 Ubiquitin-conjugating 9
Csf-1 Colony-stimulating Factor 1
VEGF Vascular endothelial growth factor
FGF-2 Fibroblast growth factor-2 


\section{References}

1. Nishimoto, S.; Nishida E. MAPK signalling: ERK5 versus ERK1/2. EMBO Rep 2006, 7: 782-786, doi: 10.1038/sj.embor.7400755.

2. Kasler, H.G.; Victoria, J.; Duramad, O.; Winoto, A. ERK5 is a novel type of mitogen-activated protein kinase containing a transcriptional activation domain. Mol Cell Biol 2000, 20: 8382-8389, doi: 10.1128/mcb.20.22.8382-8389.2000.

3. Kato, Y.: Tapping, R.I.; Huang, S.; Watson, M.H.; Ulevitch, R.J.; Lee, J.D. Bmk1/Erk5 is required for cell proliferation induced by epidermal growth factor. Nature 1998, 395: 713-716, doi: 10.1038/27234.

4. Kamakura, S.; Moriguchi, T.; Nishida, E. Activation of the protein kinase ERK5/BMK1 by receptor tyrosine kinases. Identification and characterization of a signaling pathway to the nucleus. J Biol Chem 1999, 274: 26563-26571, doi: 10.1074/jbc.274.37.26563.

5. English, J.M.; Pearson, G.; Baer, R.; Cobb, M.H. Identification of substrates and regulators of the mitogenactivated protein kinase ERK5 using chimeric protein kinases. J Biol Chem 1998, 273: 3854-3860, doi: 10.1074/jbc.273.7.3854.

6. Vaseva, A.V.; Blake, D.R.; Gilbert, T.S.K.; Ng, S.; Hostetter, G.; Azam, S.H; Ozkan-Dagliyan, I.; Gautam, P.; Bryant, K.L.; Pearce, K.H; Herring, L.E.; Han, H.; Graves, L.M.; Witkiewicz, A.K.; Knudsen, E.S.; Pecot, C.V.; Rashid, N.; Houghton, P.J.; Wennerberg, K.; Cox, A.D.; Der, C.J. KRAS Suppression-Induced Degradation of MYC Is Antagonized by a MEK5-ERK5 Compensatory Mechanism. Cancer Cell 2018, 34 : 807-822, doi: 10.1016/j.ccell.2018.10.001.

7. Zhou, G.; Bao, Z.Q.; Dixon, J.E. Components of a new human protein kinase signal transduction pathway. J Biol Chem 1995, 270: 12665-12669, doi: 10.1074/jbc.270.21.12665.

8. Lochhead, P.A.; Gilley, R.; Cook, S.J. ERK5 and its role in tumour development. Biochem Soc Trans 2012, 40: 251-256, doi: 10.1042/BST20110663.

9. Pi, X.; Garin, G.; Xie, L.; Zheng, Q.; Wei, H.; Abe, J.; Yan, C.; Berk, B.C. BMK1/ERK5 is a novel regulator of angiogenesis by destabilizing hypoxia inducible factor 1alpha. Circ Res 2005, 96: 1145-1151, doi: 10.1161/01.RES.0000168802.43528.e1.

10. Kesavan, K.; Lobel-Rice, K.; Sun, W.; Lapadat, R.; Webb, S.; Johnson, G.L.; Garrington, T.P. MEKK2 regulates the coordinate activation of ERK5 and JNK in response to FGF-2 in fibroblasts. J Cell Physiol 2004, 199: 140-148, doi: 10.1002/jcp.10457.

11. Obara, Y.; Yamauchi, A.; Takehara, S.; Nemoto, W.; Takahashi, M.; Stork, P.J.; Nakahata, N. ERK5 activity is required for nerve growth factor-induced neurite outgrowth and stabilization of tyrosine hydroxylase in PC12 cells. J Biol Chem 2009, 284: 23564-23573, doi: 10.1074/jbc.M109.027821. 
12. Rovida, E.; Spinelli, E.; Sdelci, S.; Barbetti, V.; Morandi, A.; Giuntoli, S.; Dello Sbarba, P. ERK5/BMK1 is indispensable for optimal colony-stimulating factor 1 (CSF-1)-induced proliferation in macrophages in a Src-dependent fashion. J Immunol 2008, 180: 4166-4172, doi: 10.4049/jimmunol.180.6.4166.

13. Carvajal-Vergara, X.; Tabera, S.; Montero, J.C.; Esparis-Ogando, A.; Lopez-Perez, R.; Mateo, G.; Gutierrez, N.; Parmo-Cabanas, M.; Teixido, J.; San Miguel, J.F.; Pandiella, A. Multifunctional role of Erk5 in multiple myeloma. Blood 2005, 105: 4492-4499, doi: 10.1182/blood-2004-08-2985.

14. Rovida, E.; Navari, N.; Caligiuri, A.; Dello Sbarba, P.; Marra, F. ERK5 differentially regulates PDGFinduced proliferation and migration of hepatic stellate cells. J Hepatol 2008, 48: 107-115, doi: 10.1016/j.jhep.2007.08.010.

15. Mulloy, R.; Salinas, S.; Philips, A.; Hipskind, R.A. Activation of cyclin D1 expression by the ERK5 cascade. Oncogene 2003, 22: 5387-5398, doi: 10.1038/sj.onc.1206839.

16. Yang, C.C.; Ornatsky, O.I.; McDermott, J.C.; Cruz, T.F.; Prody, C.A. Interaction of myocyte enhancer factor 2 (MEF2) with a mitogen-activated protein kinase, ERK5/BMK1. Nucleic Acids Res 1998, 26: 4771-4777, doi: 10.1093/nar/26.20.4771.

17. Kato, Y.; Kravchenko, V.V.; Tapping, R.I.; Han, J.; Ulevitch, R.J.; Lee, J.D. BMK1/ERK5 regulates seruminduced early gene expression through transcription factor MEF2C. EMBO J 1997, 16: 7054-7066, doi: 10.1093/emboj/16.23.7054.

18. Yang, Q.; Deng, X.; Lu, B.; Cameron, M.; Fearns, C.; Patricelli, M.P.; Yates JR, 3 ${ }^{\text {rd }}$; Gray, N.S.; Lee, J.D. Pharmacological inhibition of BMK1 suppresses tumor growth through promyelocytic leukemia protein. Cancer Cell 2010, 18: 258-267, doi: 10.1016/j.ccr.2010.08.008.

19. Rovida, E.; Di, M.G.; Tusa, I.; Cannito, S.; Paternostro, C.; Navari, N.; Vivoli, E.; Deng, X.; Gray, N.S.; Esparis-Ogando, A.; David, E.; Pandiella, A.; Dello Sbarba, P.; Parola, M.; Marra, F. The mitogenactivated protein kinase ERK5 regulates the development and growth of hepatocellular carcinoma. Gut 2015, 64: 1454-1465, doi: 10.1136/gutjnl-2014-306761.

20. Stecca, B.; Rovida, E. Impact of ERK5 on the Hallmarks of Cancer. Int J Mol Sci 2019, 20, doi: 10.3390/ijms20061426.

21. McCracken, S.R.; Ramsay, A.; Heer, R.; Mathers, M.E.; Jenkins, B.L.; Edwards, J, Robson CN, Marquez R, Cohen P, Leung HY. Aberrant expression of extracellular signal-regulated kinase 5 in human prostate cancer. Oncogene 2008, 27: 2978-2988, doi: 10.1038/sj.onc.1210963.

22. Montero, J.C.; Ocana, A.; Abad, M.; Ortiz-Ruiz, M.J.; Pandiella, A.; Esparis-Ogando, A. Expression of Erk5 in early stage breast cancer and association with disease free survival identifies this kinase as a potential therapeutic target. PLoS One 2009, 4: e5565, doi: 10.1371/journal.pone.0005565. 
23. Pavan, S.; Meyer-Schaller, N.; Diepenbruck, M.; Kalathur, R.K.R.; Saxena, M.; Christofori, G. A kinomewide high-content siRNA screen identifies MEK5-ERK5 signaling as critical for breast cancer cell EMT and metastasis. Oncogene 2018, 37: 4197-4213. doi: 10.1038/s41388-018-0270-8.

24. Kang, C.; Kim, J.S.; Kim, C.Y.; Kim, E.Y.; Chung, H.M. The Pharmacological Inhibition of ERK5 Enhances Apoptosis in Acute Myeloid Leukemia Cells. Int J Stem Cells 2018, 11: 227-234, doi: 10.15283/ijsc18053.

25. Mehta, P.B.; Jenkins, B.L.; McCarthy, L.; Thilak, L.; Robson, C.N.; Neal, D.E.; Leung, H.Y. MEK5 overexpression is associated with metastatic prostate cancer, and stimulates proliferation, MMP-9 expression and invasion. Oncogene 2003, 22: 1381-1389, doi: 10.1038/sj.onc.1206154.

26. Borges, J.; Pandiella, A.; Esparis-Ogando, A. Erk5 nuclear location is independent on dual phosphorylation, and favours resistance to TRAIL-induced apoptosis. Cell Signal 2007, 19: 1473-1487, doi: 10.1016/j.cellsig.2007.01.023.

27. Kondoh, K.; Terasawa, K.; Morimoto, H.; Nishida, E. Regulation of nuclear translocation of extracellular signal-regulated kinase 5 by active nuclear import and export mechanisms. Mol Cell Biol 2006, 26: 16791690, doi: 10.1128/MCB.26.5.1679-1690.2006.

28. Inesta-Vaquera, F.A.; Campbell, D.G.; Tournier, C.; Gomez, N.; Lizcano, J.M.; Cuenda, A. Alternative ERK5 regulation by phosphorylation during the cell cycle. Cell Signal 2010, 22: 1829-1837, doi: 10.1016/j.cellsig.2010.07.010.

29. Erazo, T.; Moreno, A.; Ruiz-Babot, G.; Rodriguez-Asiain, A.; Morrice, N.A.; Espadamala, J.; Bayascas, J.R.; Gomez, N.; Lizcano, J.M. Canonical and kinase activity-independent mechanisms for extracellular signalregulated kinase 5 (ERK5) nuclear translocation require dissociation of Hsp90 from the ERK5-Cdc37 complex. Mol Cell Biol 2013, 33: 1671-1686, doi: 10.1128/MCB.01246-12.

30. Stepanova, L.; Yang, G.; DeMayo, F.; Wheeler, T.M.; Finegold, M.; Thompson, T.C.; Harper, J.W. Induction of human Cdc37 in prostate cancer correlates with the ability of targeted Cdc37 expression to promote prostatic hyperplasia. Oncogene 2000, 19: 2186-2193, doi: 10.1038/sj.onc.1203561.

31. Johnson, E.S. Protein modification by SUMO. Annu Rev Biochem 2004, 73: 355-382, doi: 10.1146/annurev.biochem.73.011303.074118.

32. Tatham, M.H.; Jaffray, E.; Vaughan, O.A.; Desterro, J.M.; Botting, C.H.; Naismith, J.H.; Hay, R.T. Polymeric chains of SUMO-2 and SUMO-3 are conjugated to protein substrates by SAE1/SAE2 and Ubc9. J Biol Chem 2001, 276: 35368-35374, doi: 10.1074/jbc.M104214200.

33. Hay, R.T. SUMO-specific proteases: a twist in the tail. Trends Cell Biol 2007, 17: 370-376, doi: 10.1016/j.tcb.2007.08.002.

34. Woo, C.H.; Shishido, T.; McClain, C.; Lim, J.H.; Li, J.D.; Yang, J.; Yan, C.; Abe, J. Extracellular signalregulated kinase 5 SUMOylation antagonizes shear stress-induced antiinflammatory response and 
endothelial nitric oxide synthase expression in endothelial cells. Circ Res 2008, 102: 538-545, doi: 10.1161/CIRCRESAHA.107.156877.

35. Shishido, T.; Woo, C.H.; Ding, B.; McClain, C.; Molina, C.A.; Yan, C.; Yang, J.; Abe, J. Effects of MEK5/ERK5 association on small ubiquitin-related modification of ERK5: implications for diabetic ventricular dysfunction after myocardial infarction. Circ Res 2008, 102: 1416-1425, doi: 10.1161/CIRCRESAHA.107.168138.

36. Gomez, N.; Erazo, T.; Lizcano, J.M. ERK5 and Cell Proliferation: Nuclear Localization Is What Matters. Front Cell Dev Biol 2016, 4: 105, doi: 10.3389/fcell.2016.00105.

37. Heo, K.S.; Chang, E.; Le, N.T.; Cushman, H.; Yeh, E.T.; Fujiwara, K.; Abe, J. De-SUMOylation enzyme of sentrin/SUMO-specific protease 2 regulates disturbed flow-induced SUMOylation of ERK5 and p53 that leads to endothelial dysfunction and atherosclerosis. Circ Res 2013, 112: 911-923, doi: 10.1161/CIRCRESAHA.111.300179.

38. Drag, M.; Salvesen, G.S. DeSUMOylating enzymes--SENPs. IUBMB Life 2008, 60: 734-742, doi: 10.1002/iub.113.

39. Ramsay, A.K.; McCracken, S.R.; Soofi, M.; Fleming, J.; Yu, A.X.; Ahmad, I.; Morland, R.; Machesky, L.; Nixon, C.; Edwards, D.R.; Nuttall, R.K.; Seywright, M.; Marquez, R.; Keller, E.; Leung, H.Y. ERK5 signalling in prostate cancer promotes an invasive phenotype. Br J Cancer 2011, 104: 664-672. doi: 10.1038/sj.bjc.6606062.

40. Volkers, M.; Weidenhammer, C.; Herzog, N.; Qiu, G.; Spaich, K von WF.; Peppel, K.; Muller, O.J.; Schinkel, S.; Rabinowitz, J.E.; Hippe, H.J.; Brinks, H.; Katus, H.A.; Koch, W.J.; Eckhart, A.D.; Friedrich, O.; Most, P. The Inotropic Peptide \{beta\}ARKct Improves \{beta\}AR Responsiveness in Normal and Failing Cardiomyocytes Through G\{beta\}\{gamma\}-Mediated L-Type Calcium Current Disinhibition. Circ Res 2011, 108: 27-39, doi: 10.1161/CIRCRESAHA.110.225201.

41. Pereira, D.M.; Simoes, A.E.; Gomes, S.E.; Castro, R.E.; Carvalho, T.; Rodrigues, C.M.; Borralho, P.M. MEK5/ERK5 signaling inhibition increases colon cancer cell sensitivity to 5-fluorouracil through a p53dependent mechanism. Oncotarget 2016, 7: 34322-34340, doi: 10.18632/oncotarget.9107.

42. Sehat, B.; Tofigh, A.; Lin, Y.; Trocme, E.; Liljedahl, U.; Lagergren, J.; Larsson, O. SUMOylation mediates the nuclear translocation and signaling of the IGF-1 receptor. Sci Signal 2010, 3: ra10, doi: 10.1126/scisignal.2000628.

43. Knittle, A.M.; Helkkula, M.; Johnson, M.S.; Sundvall, M.; Elenius, K. SUMOylation regulates nuclear accumulation and signaling activity of the soluble intracellular domain of the ErbB4 receptor tyrosine kinase. J Biol Chem 2017, 292: 19890-19904, doi: 10.1074/jbc.M117.794271.

44. Wen, D.; Wu, J.; Wang, L.; Fu, Z. SUMOylation Promotes Nuclear Import and Stabilization of Polo-like Kinase 1 to Support Its Mitotic Function. Cell Rep 2017, 21: 2147-2159, doi: 10.1016/j.celrep.2017.10.085. 
45. Tomanov, K.; Nukarinen, E.; Vicente, J.; Mendiondo, G.M.; Winter, N.; Nehlin, L.; Weckwerth, W.; Holdsworth, M.J.; Teige, M.; Bachmair, A. Sumoylation and phosphorylation: hidden and overt links. J Exp Bot 2018, 69: 4583-4590, doi: 10.1093/jxb/ery167.

46. Hang, J.; Dasso, M. Association of the human SUMO-1 protease SENP2 with the nuclear pore. J Biol Chem 2002, 277: 19961-19966, doi: 10.1074/jbc.M201799200.

47. Seeler, J.S.; Dejean, A. SUMO and the robustness of cancer. Nat Rev Cancer 2017, 17: 184-197, doi: nrc.2016.143.

48. Moschos, S.J.; Jukic, D.M.; Athanassiou, C.; Bhargava, R.; Dacic, S.; Wang, X.; Kuan, S.F.; Fayewicz, S.L.; Galambos, C.; Acquafondata, M.; Dhir, R.; Becker, D. Expression analysis of Ubc9, the single small ubiquitin-like modifier (SUMO) E2 conjugating enzyme, in normal and malignant tissues. Hum Pathol 2010, 41: 1286-1298, doi: 10.1016/j.humpath.2010.02.007.

49. Hoefer, J.; Schafer, G.; Klocker, H.; Erb, H.H.; Mills, I.G.; Hengst, L.; Puhr. M.; Culig, Z. PIAS1 is increased in human prostate cancer and enhances proliferation through inhibition of p21. Am J Pathol 2012, 180: 2097-2107, doi: 10.1016/j.ajpath.2012.01.026.

50. Mody, N.; Campbell, D.G.; Morrice, N.; Peggie, M.; Cohen, P. An analysis of the phosphorylation and activation of extracellular-signal-regulated protein kinase 5 (ERK5) by mitogen-activated protein kinase kinase 5 (MKK5) in vitro. Biochem J 2003, 372: 567-575, doi: 10.1042/BJ20030193.

51. Hinz, M.; Broemer, M.; Arslan, S.C.; Otto, A.; Mueller, E.C.; Dettmer, R.; Scheidereit, C. Signal responsiveness of IkappaB kinases is determined by Cdc37-assisted transient interaction with Hsp90. J Biol Chem 2007, 282: 32311-32319, doi: 10.1074/jbc.M705785200.

52. Papapetropoulos, A.; Zhou, Z.; Gerassimou, C.; Yetik, G.; Venema, R.C.; Roussos, C.; Sessa, W.C.; Catravas, J.D. Interaction between the $90-\mathrm{kDa}$ heat shock protein and soluble guanylyl cyclase: physiological significance and mapping of the domains mediating binding. Mol Pharmacol 2005, 68: 11331141, doi: 10.1124/mol.105.012682.

53. Rodriguez-Asiain, A.; Ruiz-Babot, G.; Romero, W.; Cubi, R.; Erazo, T.; Biondi, R.M.; Bayascas, J.R.; Aguilera, J.; Gomez, N.; Gil, C.; Claro, E.; Lizcano, J.M. Brain specific kinase-1 BRSK1/SAD-B associates with lipid rafts: modulation of kinase activity by lipid environment. Biochim Biophys Acta 2011, 1811: 11241135, doi: 10.1016/j.bbalip.2011.10.004.

54. Bradford, M.M. A rapid and sensitive method for the quantitation of microgram quantities of protein utilizing the principle of protein-dye binding. Anal Biochem 1976, 72: 248-254, doi: 10.1006/abio.1976.9999.

55. Lizcano, J.M.; Alrubaie, S.; Kieloch, A.; Deak, M.; Leevers, S.J.; Alessi, D.R. Insulin-induced Drosophila S6 kinase activation requires phosphoinositide 3-kinase and protein kinase B. Biochem J 2003, 374: 297-306, doi: 10.1042/BJ20030577. 


\section{9 of 19}

56. Tatham, M.H.; Rodriguez, M.S.; Xirodimas, D.P.; Hay, R.T. Detection of protein SUMOylation in vivo. Nat Protoc 2009, 4: 1363-1371. 